\title{
Vorrang für Qualität
}

\begin{abstract}
Mieten statt Kaufen kann ein Weg zu nachhaltigerem Wirtschaften sein, der Öko-Effizienz und Suffizienz in sich vereinigt. Die Nutzer eines vermieteten Produktes verzichten auf Eigentum - das steigert die Suffizienz. Zugleich werden die Produkte intensiver genutzt, weil mehrfach hintereinander vermietet also erhöht sich die Ressourceneffizienz. Aber was kann an Vermietungskonzepten modern sein? Folgen sie nicht nach wie vor dem simplen Rezept der Leihbücherei, aus der man sich in den 50er-Jahren die Krimi-Lektüre besorgte, nur jetzt übertragen auf Werkzeuge, Autos, Sportgeräte, Elektroinstallationen?
\end{abstract}

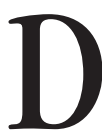
Scherborn ie Leihbücherei vermietete Bücher, deren Anschaffung man sich nicht leisten konnte; inzwischen kann man sie sich leisten, also gibt es kaum noch Leihbüchereien, ähnlich wie die Münzwaschsalons seltener geworden sind, weil die Haushalte sich ihre eigenen Waschmaschinen in die Wohnung stellen. Wenn immer nur das gemietet würde, was man sich zum Kauf nicht leisten kann, hätten Vermietungskonzepte bei wachsendem Wohlstand keine Zukunft. Sie haben Chancen, weil wir grundlegende Änderungen in Bewusstsein und Struktur erleben, und wenn die Vermieter sich auf diese Änderungen einstellen. Geändert haben sich zum einen die Werthaltungen. Die Menschen wollen heute über Dinge, die sie angehen, selbst entscheiden oder mitentscheiden. Sie wollen dabei ihre Fähigkeiten einsetzen und entfalten, kurz: Sie wollen das, was sie tun, mit innerer Anteilnahme tun, verantwortlich und kreativ. Doch sind sie häufig daran gehindert, weil - wie der Soziologe Helmut Klages betont die gesellschaftlichen Institutionen den Wandel noch nicht mitvollzogen haben, sondern Angepasstheit und Unterordnung voraussetzen (1).

Das gilt auch und gerade beim Umweltverhalten. Zu einem sehr großen Teil sind die Deutschen glaubhaft umweltbewusst. Daran ändert sich nichts, auch wenn andere Probleme vorübergehend in den Vordergrund treten - eine Umfrage des Umweltbundesamtes hat das Anfang 2000 erneut erwiesen (2). Dass das alltägliche Verhalten hinter den Einsichten zurück bleibt, hat andere Gründe. Es liegt vor allem daran, dass von den Institutionen, die das Verhalten beeinflussen, von den Preisen, Vorschriften, Organisationen und Medien, entgegengesetzte Anstöße ausgehen. Deshalb wird es nicht genügen, dass neue Ver- mietungskonzepte angeboten werden; sie müssen auch in ein infrastrukturelles Umfeld eingebettet sein, das den veränderten Werthaltungen besser Rechnung trägt.

Dass die Institutionen sich ändern müssen, hat noch einen weiteren Grund. Denn zum anderen wandelt sich auch die Wirtschaftsstruktur. Wir sind mit Wirtschaftstätigkeiten und Produkten in die Informations- und Dienstleistungsgesellschaft eingetreten. Zwei Drittel der Erwerbstätigkeiten sind inzwischen Dienstleistungen, die unbezahlten Tätigkeiten sind es ohnehin zum größten Teil. Unser Bewusstsein aber orientiert sich noch an den Kategorien der Industriegesellschaft.

\section{- Neue Vermietungskonzepte}

Im industriellen Kontext besteht das Vermieten darin, Industrieprodukte für eine Zeitspanne gegen Entgelt zur Verfügung zu stellen. In der Informations- und Dienstleistungsgesellschaft dagegen muss der Vermieter mehr zur Verfügung stellen als ein Industrieprodukt. Denn nicht nur die Produkte sind komplexer, weil mehr Informationen und Dienstleistungen in sie inkorporiert

Integrierte
Produktpolitik
ist das Thema
des Informationsdienstes
Ökologisches Wirtschaften 6/00
Wenn Sie potenzielle Beiträge
haben, wenden Sie sich bitte
an die Redaktion.

sind; auch das Vermieten selbst ist es. Vermietet wird nun ein Komplex von Dienstleistungen. Dabei kann man zwei Möglichkeiten unterscheiden:

Wo das Produkt - wie etwa eine Heizanlage vom Nutzer passiv genutzt wird, da wird nicht mehr das Produkt vermietet, sondern die von ihm erwartete Dienstleistung. Diese wird mit der jeweils größtmöglichen Effizienz hervorgebracht, also mit den jeweils effizientesten Verfahren und Produkten. Der Vermieter nimmt dem Nutzer die Investitionsentscheidungen und -aufwendungen $\mathrm{ab}$, erbringt also auch selbst eine Dienstleistung, die über das Vermieten eines Produkts hinausgeht.

- Wo die Nutzung des Produkts - wie etwa einer Computersoftware - in eigenen Aktivitäten der Nutzer besteht, da stellt der Vermieter die Voraussetzungen dafür bereit, dass diese Aktivitäten so effizient wie möglich ausgefiuhrt werden können, beispielsweise indem er dem Nutzer die jeweils modernste und effizienteste Software zur Verfiigung stellt und ihm die Unterhaltung umfangreicher Hardware abnimmt; in naher Zukunft wird man auch die Speicherkapazität im Netz anmieten können.

In beiden Fällen wird den Nutzern durch die Vermietungsleistung weit mehr abgenommen als der Kauf von Produkten. Sie sparen daneben einen beträchtlichen Aufwand an Zeit und psychischer Energie für den Erwerb und die Weiterentwicklung des erforderlichen Wissens ein. Ihre Leistung ist in solchem Maße eine Dienstleistung, dass auf sie auch der industrielle Produktivitätsbegriff nicht mehr ohne weiteres zutrifft.

Bei der bloßen Vermietung von Produkten wird die Produktivität nach der Anzahl der pro Zeiteinheit abgeschlossenen Mietverträge bemessen - je weniger Zeit der Vermieter für einen Vertrag aufwändet, desto produktiver und einträglicher ist seine Tätigkeit. Für die Steigerung der Produktivität kommt es also darauf an, den Zeitaufwand pro Vermietung zu verringern. Die Qualität der neuen Vermietungsleistungen ist aber sehr sensibel, weil sie wesentlich von der aufgewandten Zeit abhängig ist. Die Qualität leidet, wenn die Zeit unter ein optimales Maß verkürzt wird. Daher kann das Denkmuster der industriellen Produktivitätssteigerung nicht mechanisch übertragen werden; für die Produktivität der Dienstleistungen spielt die Qualität eine weit größere Rolle. In dem Quotienten „Leistung dividiert durch Einsatz" ist die Möglichkeit, den Arbeitseinsatz im Nenner zu verringern oder bei gleichbleibendem Einsatz die Anzahl oder den Umfang der Leistun- 
gen zu erhöhen, sehr begrenzt - die Produktivität ist eher dadurch zu steigern, dass die Qualität der Leistung im Zähler verbessert wird.

\section{- Qualität versus Eigentum}

Hier liegt die Herausforderung, die von den neuen Vermietungskonzepten ausgeht. Die potenziellen Vermieter müssen begreifen, dass sie nicht Produkte vermieten, sondern eine Kombination von Dienstleistungen, die teils von den Produkten und teils von ihnen selbst hervorgebracht werden. Das vermietete Gesamtpaket erhält seine besondere Qualität dadurch, dass die Vermieter es besser an die Anforderungen der Mieter und an den Wandel der äußeren Gegebenheiten anpassen können als es den Mietern mit ihren begrenzten Kapazitäten möglich wäre. Diese Qualität hat ihren Preis, weil sie einen hohen Investitionsaufwand voraussetzt, und weil sie nur mit beträchtlichem Zeitaufwand aufrechterhalten werden kann. Deshalb wird sich die Leistung am Markt weniger durch Senkung des Preises behaupten, als vielmehr durch Wahrung und Weiterentwicklung der Qualität. Die allerdings wird nachgewiesen und geprïft werden müssen, gerade wenn sie hohe Preisforderungen rechtfertigen soll.

Auf der anderen Seite müssen die Mieter begreifen, dass sie für den Verzicht auf Eigentum ein Leistungspaket eintauschen, das sie selbst, durch den Einsatz eigener Produkte und eigenen Wissens, nicht so effizient hervorbringen könnten, und dass dieser Vorteil die Kosten aufwiegt, die im Preis der Leistung und im Verzicht auf das Eigentum liegen. Das zu begreifen, wird vielen nicht leicht fallen, weil sie mit dem Eigentum an Sachen Gefühle von Verfügungsfreiheit und Macht verbinden, die für manchen schwer wiegen, zumal wenn sie auch mit einem vermeintlichen Vorsprung an Komfort verbunden sind. All das muss durch den Qualitätsvorsprung der Vermietungskonzepte aufgewogen werden - eine große Aufgabe, die nicht schnell und nicht überall erfïllbar sein wird.

Dass sie heute erfüllbar scheint, darin liegt eine große Hoffnung. Denn zu den Hindernissen für nachhaltiges Wirtschaften gehört die übermäßige Präferenz für das Privateigentum an Produktionsmitteln, im Unternehmen wie im privaten Haushalt. Wenn diese Vorliebe auf ein vernünftiges Maß zurückgefuihrt wird, ist viel gewonnen. Die neuen Vermietungskonzepte können dabei helfen, zumal wenn sie im Zusammenhang mit steigenden Kosten für fossile Energien, nichterneuerbare Rohstoffe und schädliche Emissionen stehen.
Denn dann sind die beiden Gefahren gering, die man ihnen in einem Umfeld zuschreiben muss, in dem die gesellschaftlichen Institutionen weiter an Naturausbeutung und -zerstörung orientiert blieben. Die erste besteht darin, dass das den kommerziellen Vermietungskonzepten innewohnende Innovationsmoment den Energie- und Stoffdurchsatz eher noch erhöhen könnte, weil die vermietenden Unternehmen den Investitionszyklus verkürzen, um stets das Neueste anbieten zu können. Im Kontext einer umweltschonenden Ordnungspolitik aber wird dieses Innovationsmoment den Energie- und Stoffdurchsatz eher senken, weil die Wiederverwendung stärker belohnt wird.

\section{Verdrängung nichłkommerzieller Konzepte?}

Die zweite Gefahr kann man darin sehen, dass der Erfolg der kommerziellen Vermietungskonzepte die nichterwerbswirtschaftlichen Initiativen zum Tauschen, Leihen und Teilen von Produkten verdrängen könnte, weil diese im Vergleich der Leistungen und Kosten nicht bestehen. Im Kontext einer umweltschonenden Ordnungspolitik aber ist zu erwarten, dass der Erfolg der Vermietungskonzepte die nichtkommerziellen Aktivitäten eher beflügelt. Im Bereich der arbeitsintensiven Dienstleistungen werden kommerzielle Vermietungskonzepte weniger rentabel sein als dort, wo das Produkt und die Dienstleistung vom Computer übernommen werden. Ein Beispiel ist, dass man den Anrufbeantworter nicht mehr kauft, sondern seine Leistung aus dem Netz bezieht und dafür Gebühren zahlt. So wird es nicht nur mehr kommerzielle Vermietungskonzepte geben; auch das Selbermachen und die gemeinschaftliche Produktion werden angeregt, weil sie vom Markt unabhängig sind.

Umgekehrt beziehen ja auch sie selbst aus diesem Bereich immer wieder Anregungen. Heute bietet vor allem die Computertechnik instruktive Beispiele dafür, dass das kommerzielle und das nichtkommerzielle Handeln einander fruchtbar ergänzen können. Auch das Car-Sharing hat neben der Autovermietung seinen eigenen Platz. Wir haben es hier mit zwei unterschiedlichen Konzepten - Vermieten und Teilen - zu tun, die nebeneinander existieren können und gleichwohl in gewisser Konkurrenz zueinander stehen, weil ihre Leistungen mit denen des anderen Konzepts verglichen werden.

Um den Vergleich immer wieder aushalten zu können, müssen sie sich weiterentwickeln. Das ist ein Prozess, dessen weitere Stationen man aufmerksam begleiten muss. Im Falle der Autovermietung und des Car-Sharing dürfte er zur Entwicklung einer kommunikationstechnischen Infrastruktur der temporären Autonutzung führen, die den Charakter eines öffentlichen Gutes mit bedingtem Zugang hat, und es wird eine spannende Frage sein, ob sich divergierende Lösungen entwickeln oder ob es eine Lösung geben kann, die Beidem, dem Mieten wie dem Teilen von Autos, auf je eigene Weise zugute kommt. Diese Ambivalenz des Wandels zur Dienstleistungsgesellschaft nimmt auch Rifkin in seinem neuen Buch auf (3): Er argumentiert, dass wir auf eine Wirtschaftsepoche zusteuern, in der die Märkte den Netzwerken Platz machen und das Eigentum durch den „Zugang zu Erfahrungen“ (access to experiences) ersetzt wird, für den man zahlt. Miete ist eine Form des Zugangs zu Produkten und Dienstleistungen, andere Formen sind das nichtkommerzielle Leihen, Teilen, Tauschen, das Selbermachen, die Nachbarschaftshilfe, die Gemeinschaftsarbeit. Sie werden in der neuen Ära ebenso Aufwind bekommen wie die Vermietungskonzepte, nämlich überall dort, wo diese zu teuer sind oder ihre Qualität nicht befriedigt.

\section{Anmerkungen}

(1) Vgl. den Beitrag von H. Klages in Klages, Helmut/ Gensicke, Thomas "Wertewandel und bürgerschaftliches Engagement an der Schwelle zum 21. Jahrhundert". Forschungsinstitut für Öffentliche Verwaltung, Speyer 1999.

(2) Vgl. Kuckartz, Udo: Umweltbewusstsein in Deutschland 2000 - Ergebnisse einer repräsentativen Bevölkerungsumfrage im Auftrag des Umweltbundesamtes. Im Internet unter: www.umweltbewusstsein.de

(3) Rifkin, Jeremy: The age of access. The new culture of hypercapitalism where all of life is a paid-for experience, New York 2000; deutsch: Access. Das Verschwinden des Eigentums. Wenn alles im Leben zur bezahlten Ware wird, Frankfurt/M. 2000.

\section{Der Autor}

Dr. Gerhard Scherhorn ist emeritierter Professor für Konsumökonomik an der Universität Hohenheim, Stuttgart, und Direktor der Arbeitsgruppe Neue Wohlstandsmodelle im Wuppertal Institut für Klima,

Umwelt und Energie.

Kontakt: Wuppertal Institut, Postfach 100480 ,

42004 Wuppertal,

E-mail: gerhard.scherhorn@wupperinst.org 
(c) 20I0 Authors; licensee IÖW and oekom verlag. This is an article distributed under the terms of the Creative Commons Attribution Non-Commercial No Derivates License (http://creativecommons.org/licenses/by-nc-nd/3.o/), which permits unrestricted use, distribution, and reproduction in any medium, provided the original work is properly cited. 